\title{
A CLASSIFICATION OF KERNELS WHICH POSSESS INTEGRAL TRANSFORMS
}

\section{CHARLES FOX}

1. Introduction. Consider the equation

$$
f(x)=\int_{0}^{\infty} k(u x) g(u) d u,
$$

where $f(x)$ and $k(x)$ are known functions and where we are required to determine the function $g(x)$. The purpose of this paper is to investigate the conditions under which this problem can be solved by means of an inversion formula in the nature of an integral transform. An example of such an inversion formula occurs in the theory of Fourier Transforms. I shall prove that the Mellin transform of $k(x)$ can be classified in such a manner that with each class can be associated a particular type of integral transform.

The two functions $f(u)$ and $F(s)$ are said to be Mellin transforms of each other if they are related in the following manner:

$$
F(s)=\int_{0}^{\infty} f(u) u^{s-1} d u .
$$

Associated with (2) is the reciprocal formula

$$
f(u)=\frac{1}{2 \pi i} \int_{c-i \infty}^{c+i \infty} F(s) u^{-s} d s .
$$

Here $c$ is a real constant and we shall always take $c=1 / 2$. All pairs of functions written in this form, $f(u), F(s) ; g(u), G(s) ; k(u), K(s)$; etc. will, in this paper, be Mellin transforms of each other and will be related as $f(u)$ and $F(s)$ are in (2) and (3). We shall frequently write $s=1 / 2+i t$, so that, in the integral of (3), $t$ will vary from $-\infty$ to $+\infty$. We shall also refer to $k(x)$ as a kernel.

In order to illustrate the results obtained later we shall first proceed formally. Multiply (1) by $x^{8-1}$ and integrate with respect to $x$ from 0 to $\infty$. The double integral on the right-hand side can be evaluated by writing $x=v / u$ and we then find that

$$
F(s)=K(s) G(1-s) \text {. }
$$

This is equivalent to

Presented to the Society, April 20, 1956; received by the editors June 9, 1955. 


$$
G(s)=\frac{K(s) F(1-s)}{K(s) K(1-s)} .
$$

Now in one of the classifications to be discussed later on $K(s)$ will satisfy the functional equation

$$
K(s) K(1-s)=\frac{1}{a+b s(1-s)},
$$

where $a$ and $b$ are constants. From (5) and (6) we now deduce

$$
G(s)=a K(s) F(1-s)+b s(1-s) K(s) F(1-s) .
$$

On multiplying (7) by $x^{-s}$, and, integrating along the line $s=1 / 2+i t$, from $t=-\infty$ to $+\infty$, and then using formulae related to (3) we obtain the following result:

$$
g(x)=a \int_{0}^{\infty} k(u x) f(u) d u+b \frac{d}{d x} \int_{0}^{\infty} x k(u x) \frac{d}{d u}\{u f(u)\} d u .
$$

We have thus solved (1), regarded as an equation with $g(u)$ as an unknown function, by a method akin to the theory of Fourier Transforms, Titchmarsh [1, Chapter 8]. In fact (1) and (8) reduce to a pair of Fourier transforms in the case when $b=0$.

To give a simple illustration of this result when $b \neq 0$ consider the case when

$$
K(s)=\frac{1}{a+s},
$$

where $a$ is a constant. Evidently

$$
K(s) K(1-s)=\frac{1}{a^{2}+a+s(1-s)}
$$

so that (6) is satisfied. It is easy to verify from (2) that $K(s)$ defined in (9) is the Mellin transform of $k(u)=u^{a}(0<u<1), k(u)=0(u>1)$. Equation (1) then becomes

$$
f(x)=\int_{0}^{1 / x} u^{a} x^{a} g(u) d u,
$$

and the inversion formula ( 8 ) becomes

(12) $g(x)=\left(a^{2}+a\right) \int_{0}^{1 / x} u^{a} x^{a} g(u) d u+\frac{d}{d x} \int_{0}^{1 / x} u^{a} x^{a+1} \frac{d}{d u}\{u f(u)\} d u$. 
On integrating the second integral of (12) by parts and then differentiating, (12) reduces to

$$
g(x)=\frac{a}{x} f\left(\frac{1}{x}\right)-\frac{1}{x^{2}} f^{\prime}\left(\frac{1}{x}\right) .
$$

It is now easily verified that (11) and (13) are essentially the same equation. Another example is given by (60), $\$ 7$.

In $\$ 2$ we catalogue some properties of Mellin transforms needed for the proof of (8). In $\$ \S 3$ and 4 we shall prove (8) and some closely related theorems. In $\$ \S 5$ and 6 we shall establish integral transforms which are symmetrical in form, unlike (1) and (8) which are obviously unsymmetrical. In $\S 7$ we shall discuss the classification of $K(s)$ which gives rise to integrals such as (1) and (8).

2. Properties of Mellin transforms. A. (Titchmarsh [1, §3.17].) If $f(u) \in L^{2}(0, \infty)$ then

$$
\underset{a \rightarrow \infty}{\lim .} \int_{1 / a}^{a} f(u) u^{s-1} d u=F(s),
$$

where l.i.m. denotes, as usual, the limit in mean square. (14) asserts that l.i.m. of the left-hand side exists and that this limit is denoted by $F(s)$. Furthermore $F(s)$ possesses the following properties: $F(s) \in L^{2}(1 / 2-i \infty, 1 / 2+i \infty)$ and

$$
\text { li.i.m. } \frac{1}{2 \pi i} \int_{1 / 2-i a}^{1 / 2+i a} F(s) u^{-s} d s=f(u) \text {. }
$$

Conversely, if $F(s) \in L^{2}(1 / 2-i \infty, 1 / 2+i \infty)$, then the limit in (15) exists and defines a function $f(u)$ with the following properties: $f(u) \in L^{2}(0, \infty)$ and $(14)$ is true.

In future we shall write $f(u) \in L^{2}$ and $F(s) \in L^{2}$ where it is to be understood that for a function denoted by a small letter, such as $f$, the range of integration is from 0 to $\infty$ and for a function denoted by a capital letter, such as $F$, the range is from $1 / 2-i \infty$ to $1 / 2+i \infty$.

B. (Titchmarsh $[1, \S 3.17]$.) If either $f(u) \in L^{2}$ and $g(u) \in L^{2}$, or $F(s) \in L^{2}$ and $G(s) \in L^{2}$, then

$$
\int_{0}^{\infty} f(u) g(u) d u=\frac{1}{2 \pi i} \int_{1 / 2-i \infty}^{1 / 2+i \infty} F(s) G(1-s) d s .
$$

We shall use (16) in a slightly different form. If $f(u) \in L^{2}$ then, considered as a function of $u$, we also have $f(u x) \in L^{2}$. Also, from (2) or (3), if $F(s)$ is the Mellin transform of $f(u)$ then $F(s) x^{-8}$ is the 
Mellin transform of $f(u x)$. Hence (16) can be written in the form

$$
\int_{0}^{\infty} f(u x) g(u) d u=\frac{1}{2 \pi i} \int_{1 / 2-i \infty}^{1 / 2+i \infty} F(s) G(1-s) x^{-s} d s .
$$

3. Theorem 1. If (i) on the line $s=1 / 2+i t \quad(-\infty<t<\infty)$, $\left|G(s) s^{c+1}\right|(c>0)$ and $|H(s)|$ are both bounded; (ii)

$$
K(s)=H(s) /(m+n s),
$$

where $m$ and $n$ are constants and $K(s)$ has no poles on the line $s=1 / 2+i t$; (iii) $H(s) H(1-s)=1$ and (iv)

$$
f(x)=\int_{0}^{\infty} k(u x) g(u) d u
$$

then $f(u) \in L^{2}$ and

$$
g(x)=a \int_{0}^{\infty} k(u x) f(u) d u+b \frac{d}{d x} \int_{0}^{\infty} x k(u x) \frac{d}{d u}\{u f(u)\} d u
$$

where $a=m^{2}+m n$ and $b=n^{2}$.

Proof. From (i) and (ii), $G(s) \in L^{2}$ and $K(s) \in L^{2}$. Hence, from $B, \S 2$, it follows that the integral in (18) exists and that we have

$$
f(x)=\frac{1}{2 \pi i} \int_{1 / 2-i \infty}^{1 / 2+i \infty} K(s) G(1-s) x^{-s} d s .
$$

Now it is evident from (i) and (ii) that $K(s) G(1-s) \in L^{2}$, hence from $A, \$ 2$ we conclude that

$$
F(s)=K(s) G(1-s) .
$$

Again from (ii) and (iii) we have

$$
\begin{aligned}
K(s) K(1-s) & =\frac{1}{(m+n s)(m+n-n s)} \\
& =\frac{1}{a+b s(1-s)}
\end{aligned}
$$

where $a=m^{2}+m n$ and $b=n^{2}$. Hence, from (21) we have

$$
\begin{aligned}
G(s) & =\frac{F(1-s)}{K(1-s)}=\frac{K(s) F(1-s)}{K(s) K(1-s)} \\
& =a K(s) F(1-s)+b K(s) F(1-s) s(1-s) .
\end{aligned}
$$


But, from (i), (ii) and (21), we can deduce that on the line

$$
s=\frac{1}{2}+i t,
$$

$\left|F(s) s^{c+2}\right|(c>0)$ is bounded. Hence all the integrals in the following equation converge and so from (25) it follows that

$$
\begin{aligned}
\frac{d}{d x} \int_{1 / 2-i \infty}^{1 / 2+i \infty} \frac{G(s)}{1-s} x^{1-s} d s= & a \frac{d}{d x} \int_{1 / 2-i \infty}^{1 / 2+i \infty} \frac{K(s)}{1-s} F(1-s) x^{1-s} d s \\
& +b \frac{d}{d x} \int_{1 / 2-i \infty}^{1 / 2+i \infty} K(s) F(1-s) s x^{1-s} d s
\end{aligned}
$$

We shall complete the proof by showing that (26) and (19) are the same equation.

Dealing first with the term on the left of (26) we have, from (i), $\int_{1 / 2-i \infty}^{1 / 2+i \infty} G(s) x^{-8} d s$ is uniformly convergent with respect to $x$, for any $x$-interval which excludes the value $x=0$. Hence

$$
\begin{aligned}
\frac{d}{d x} \int_{1 / 2-i \infty}^{1 / 2+i \infty} \frac{G(s)}{1-s} x^{1-s} d s & =\int_{1 / 2-i \infty}^{1 / 2+i \infty} G(s) x^{-8} d s \\
& =2 \pi i g(x),
\end{aligned}
$$

on using A, $\$ 2$, since $G(s) \in L^{2}$.

Dealing with the first term on the right of (26) we have already shown that on the line $s=1 / 2+i t,\left|F(s) s^{c+2}\right|(c>0)$ is bounded. Coupling this with conditions (i) and (ii) we see that $\int_{1 / 2-1 \infty}^{1 / 2+\infty} K(s) F(1-s) x^{-8} d s$ is uniformly convergent with respect to $x$ in any interval which does not include $x=0$. Hence

$$
\begin{aligned}
\frac{d}{d x} \int_{1 / 2-i \infty}^{1 / 2+i \infty} \frac{K(s)}{1-s} F(1-s) x^{1-s} d s & =\int_{1 / 2-i \infty}^{1 / 2+i \infty} K(s) F(1-s) x^{-s} d s \\
& =2 \pi i \int_{0}^{\infty} k(u x) f(u) d u
\end{aligned}
$$

on using $\mathrm{B}, \S 2$. The use of $\mathrm{B}, \S 2$ is justified since it is evident from the behaviour of $K(s)$ and $F(s)$ on the line $s=1 / 2+i t$ that both these functions belong to $L^{2}$.

The last term in (26) is dealt with in the same way but presents a little more difficulty. It is evident from the fact that $\left|F(s) s^{c+2}\right|(c>0)$ is bounded on the line $s=1 / 2+i t$ that $F(s) \in L^{2}$ and that the integral of (32) below is uniformly convergent with respect to $u$, for any $u$-interval which does not include $u=0$. Hence 


$$
\begin{aligned}
\frac{d}{d u}\{u f(u)\} & =\frac{d}{d u} \frac{1}{2 \pi i} \int_{1 / 2-i \infty}^{1 / 2+i \infty} F(s) u^{1-s} d s \\
& =\frac{1}{2 \pi i} \int_{1 / 2-i \infty}^{1 / 2+i \infty} F(s)(1-s) u^{-s} d s .
\end{aligned}
$$

Consequently the Mellin transform of $d\{u f(u)\} / d u$ is $F(s)(1-s)$. From the boundedness of $\left|F(s) s^{c+2}\right|(c>0)$ on the line $s=1 / 2+i t$ it follows that $F(s)(1-s) \in L^{2}$ and since $K(s) \in L^{2}$ we deduce from $\mathrm{B}, \S 2$ that

$$
\begin{aligned}
\frac{d}{d x} \int_{1 / 2-i \infty}^{1 / 2+i \infty} K(s) F(1-s) s x^{1-s} d s & \\
& =2 \pi i \frac{d}{d x} \int_{0}^{\infty} k(u x) x \frac{d}{d u}\{u(f u)\} d u .
\end{aligned}
$$

On comparing the equations (19) and (26) term by term we see from (28), (30), and (33) that these two equations are the same. This completes the proof.

4. Theorem 1a. This is the converse of Theorem 1. If (i) on the line $s=1 / 2+i t(-\infty<t<\infty),\left|F(s) s^{c+2}\right|(c>0)$, and $H(s)$ are both bounded; (ii) and (iii) as in Theorem 1 both hold then equation (19) implies that $g(x) \in L^{2}$ and (18) is true.

These conditions enable us to apply the arguments of theorem 1 in reverse order and so it is not necessary to give the proof in detail.

TheOREM 1b. Let $f_{1}(x)$ and $g_{1}(x)$ be related in the same manner as $f(x)$ and $g(x)$ are in (18). Also let $f_{2}(x)$ and $g_{2}(x)$ be similarly related. Then, if $G_{1}(s)$ and $G_{2}(s)$ both satisfy the same conditions as $G(s)$ does in Theorem 1 and $K(s)$ satisfies the same conditions as $K(s)$ does in Theorem 1, we have

$$
\int_{0}^{\infty} f_{1}(u x) g_{2}(u) d u=\int_{0}^{\infty} f_{2}(u x) g_{1}(u) d u .
$$

Proof. The methods used in the proof of Theorem 1 to establish (21) can be used here to establish the following results:

$$
\begin{aligned}
& F_{1}(s)=K(s) G_{1}(1-s), \\
& F_{2}(s)=K(s) G_{2}(1-s) .
\end{aligned}
$$

These methods also enable us to state that all the functions of (35) and (36) belong to $L^{2}$.

Evidently from (35) and (36) we have 


$$
F_{1}(s) G_{2}(1-s)=F_{2}(s) G_{1}(1-s) .
$$

On multiplying (37) by $x^{-s}$ and integrating with respect to $s$ from $1 / 2-i \infty$ to $1 / 2+i \infty$ we may then apply (17) to the result so obtained. The final conclusion is then the equation (34) above.

5. A symmetrical inversion formula. In this section we shall deal with the case when

$$
K(s)=\frac{H(s)}{a+b s(1-s)}
$$

where, as previously,

$$
H(s) H(1-s)=1
$$

We then obtain a theorem which is symmetrical in form and which closely resembles the Generalized Fourier Transform (Titchmarsh [1, Chap. 8]).

TheOREM 2. If (i) on the line $s=1 / 2+i t(-\infty<t<\infty),\left|G(s) s^{c+2}\right|$ $(c>0)$ and $|H(s)|$ are both bounded; (ii) $K(s)=H(s) /\{a+b s(1-s)\}$, where $a$ and $b$ are constants and $K(s)$ has no poles on the line $s=1 / 2+i t$; (iii) $H(s) H(1-s)=1$; and (iv)

$$
f(x)=a \int_{0}^{\infty} k(u x) g(u) d u+b \frac{d}{d x} \int_{0}^{\infty} k(u x) x \frac{d}{d u}\{u g(u)\} d u
$$

then $f(x) \in L^{2}$ and

$$
g(x)=a \int_{0}^{\infty} k(u x) f(u) d u+b \frac{d}{d x} \int_{0}^{\infty} k(u x) x \frac{d}{d u}\{u f(u)\} d u .
$$

Proof. The proof of Theorem 2 proceeds on much the same lines as that of Theorem 1a, the converse of Theorem 1 .

From (i) and (ii), $G(s) \in L^{2}$ and $K(s) \in L^{2}$ and the integral in (43) below is uniformly convergent with respect to $u$, (in any interval which excludes $u=0$ ). Hence

$$
\begin{aligned}
\frac{d}{d u}\{u g(u)\} & =\frac{d}{d u} \frac{1}{2 \pi i} \int_{1 / 2-i \infty}^{1 / 2+i \infty} G(s) u^{1-s} d s \\
& =\frac{1}{2 \pi i} \int_{1 / 2-i \infty}^{1 / 2+i \infty} G(s)(1-s) u^{-s} d s .
\end{aligned}
$$

Evidently, from (i), $G(s)(1-s) \in L^{2}$, hence by A, $\$ 2$ and (43) the Mellin transform of $d\{u g(u)\} / d u$ is $G(s)(1-s)$. Also $K(s) \in L^{2}$, hence from (17) B, \$2 we have 


$$
\int_{0}^{\infty} k(u x) \frac{d}{d u}\{u g(u)\} d u=\frac{1}{2 \pi i} \int_{1 / 2-i \infty}^{1 / 2+i \infty} K(s) G(1-s) s x^{-8} d s .
$$

Finally from (i) and (ii), $\left|K(s) G(1-s) s^{c+4}\right|(c>0)$ is bounded on the line $s=1 / 2+i$ and so, from uniform convergence of the integral on the right-hand side of (45) below, we may deduce from (44) that

$$
\begin{aligned}
\frac{d}{d x} \int_{0}^{\infty} k(u x) x \frac{d}{d u}\{u g(u)\} & d u \\
& =\frac{1}{2 \pi i} \int_{1 / 2-i \infty}^{1 / 2+i \infty} K(s) G(1-s) s(1-s) x^{-8} d s
\end{aligned}
$$

Again, since $K(s)$ and $G(s)$ both belong to $L^{2}$, from (17), B, $\$ 2$, we have

$$
\int_{0}^{\infty} k(u x) g(u) d u=\frac{1}{2 \pi i} \int_{1 / 2-i \infty}^{1 / 2+i \infty} K(s) G(1-s) x^{-8} d s .
$$

Comparison of (45) and (46) with (40) now shows that the integrals on the right-hand side of $(40)$ exist and that

$$
f(x)=\frac{1}{2 \pi i} \int_{1 / 2-i \infty}^{1 / 2+i \infty} K(s) G(1-s)\{a+b s(1-s)\} x^{-s} d s .
$$

But, from (i) and (ii), $\left|K(s) G(1-s)\{a+b s(1-s)\} s^{c+2}\right|(c>0)$ is bounded on the line $s=1 / 2+i t$ and so the integrand on the righthand side of (47), as a function of $s$, belongs to $L^{2}$. Hence, from A, $\$ 2$ and (47) we have

$$
F(s)=K(s) G(1-s)\{a+b s(1-s)\} .
$$

Finally, from (i), (ii) and (48) we conclude that $\left|F(s) s^{c+2}\right|(c>0)$ is also bounded on the line $s=1 / 2+i t$.

Therefore the integrals on the right-hand side of (41) must exist. As we cannot assume that equation (41) is true let us denote the right-hand side by $p(x)$.

Since we have just proved that $F(s)$ satisfies the same condition as that imposed on $G(s)$, it follows that we may deal with the righthand side of (41) in exactly the same manner as we have dealt with the right-hand side of $(40)$, i.e. we may replace $f(x)$ by $p(x)$ and $g(u)$ by $f(u)$ in all the preceding arguments. Instead of (48) we then get

$$
P(s)=K(s) F(1-s)\{a+b s(1-s)\} .
$$

Now from (ii) and (iii) we have 


$$
K(s) K(1-s)\{a+b s(1-s)\}^{2}=1 .
$$

Hence, on replacing $s$ by $(1-s)$ in (48), multiplying the result by (49) and using (50) we obtain

$$
P(s)=G(s) .
$$

Finally, since $G(s) \in L^{2}$ (and so therefore $P(s) \in L^{2}$ also) it follows from $A, \S 2$ that

$$
p(x)=g(x)
$$

almost everywhere. This evidently establishes (41) and so completes the proof of Theorem 2.

6. Theorem 2a (Analogue of Parseval's Theorem). Let $f_{1}(x)$ and $g_{1}(x)$ be related as $f(x)$ and $g(x)$ are in equation (40). Let $f_{2}(x)$ and $g_{2}(x)$ be similarly related with the same kernel $k(x)$ as is used for $f_{1}(x)$ and $g_{1}(x)$. If $G_{1}(s)$ and $G_{2}(s)$ each satisfy the condition imposed upon $G(s)$ in Theorem 2 and if $K(s)$ also satisfies the conditions of that theorem then

$$
\int_{0}^{\infty} f_{1}(u x) f_{2}(u) d u=\int_{0}^{\infty} g_{1}(u) g_{2}(u x) d u
$$

Proof. By the same arguments as are used in the proof of Theorem 2 to deduce equation (48) we can prove that

$$
F_{1}(s)=K(s) G_{1}(1-s)\{a+b s(1-s)\}
$$

$$
F_{2}(s)=K(s) G_{2}(1-s)\{a+b s(1-s)\} .
$$

Hence, on replacing $s$ by $(1-s)$ in (55), multiplying the result by (55) and using (50) we deduce that

$$
F_{1}(s) F_{2}(1-s)=G_{1}(1-s) G_{2}(s) .
$$

Also, as in the proof of Theorem 2, we can show that each of the factors in (56) belong to $L^{2}$.

Now multiply both sides of (56) by $x^{-s}$ and integrate with respect to $s$ from $1 / 2-i \infty$ to $1 / 2+i \infty$. On applying (17), $\mathrm{B}, \S 2$ to the result so obtained we immediately establish equation (53) and so complete the proof of Theorem $2 \mathrm{a}$. If we divide (54) by (55) we can obtain a result identical with (34). Owing to the symmetry of Theorem 2, $f(x)$ and $g(x)$ can be interchanged, and so, for this case, (34) and (53) are essentially the same. For Theorem 1, owing to the unsymmetrical nature of equations (18) and (19) a result such as (53) does not hold. 
7. A kernel classification. In this section we shall discuss the generation and classification of kernels $k(x)$ such as are required for use with Theorems 1 and 2.

The first class of kernels will be designated by the subscript zero. The function $k_{0}(x)$ will belong to this class if its Mellin transform $K_{0}(s)$ is bounded on the line $s=1 / 2+i t$ and also satisfies the functional equation

$$
K_{0}(s) K_{0}(1-s)=1 .
$$

In this case $k_{0}(x)$ is the kernel of a generalized Fourier Transform (Titchmarsh [1, Chapter 8], Hardy and Titchmarsh [2], Watson [3], Paley and Wiener [4]). Large classes of such functions are well known.

The second class of kernels will be designated by the subscript one. The function $k_{1}(x)$ will belong to this class if its Mellin transform $K_{1}(s)$ is of the form

$$
K_{1}(s)=K_{0}(s) /(m+n s)
$$

where $K_{0}(s)$ satisfies the conditions imposed upon the Mellin transforms of functions of class $k_{0}(x)$ above. We shall also assume that $K_{1}(s)$ has no poles on the line $s=1 / 2+i t$.

Since $K_{0}(s)$ is bounded on the line $s=1 / 2+i t$, it follows that $K_{0}(s) /(m+n s) \in L^{2}$. Hence

$$
k_{1}(x)=\text { l.i.m. } \frac{1}{2 \pi i} \int_{1 / 2-i \infty}^{1 / 2+i \infty} \frac{K_{0}(s)}{m+n s} d s
$$

exists. Again, since $K_{0}(s) K_{0}(1-s)=1$, conditions (i), (ii) and (iii) of Theorem 1 are satisfied and consequently $k_{1}(x)$ is a kernel which can be used in the equations of this theorem.

In the example given in $\S 1$, equation (9) we made $K_{0}(s)=1$ and $m=a, n=1$.

Another example is given by taking $\pi^{1 / 2} k_{0}(x)=2^{1 / 2} \cos x$, $(\pi / 2)^{1 / 2} K_{0}(s)=\Gamma(s) \cos (s \pi / 2)$, and $K_{1}(s)=K_{0}(s) /(1+s)$. The integral of (59) may then be evaluated by means of the poles of $K_{1}(s)$, all of which lie on the left of the line of integration. We then obtain the following result

(60) $\left(\frac{\pi}{2}\right)^{1 / 2} k_{1}(x)=-\frac{1}{2} \pi x+\sum_{n=0}^{\infty} \frac{(-1)^{n} x^{2 n}}{(2 n) !(1-2 n)}=x \int_{x}^{\infty} \frac{\cos u}{u^{2}} d u$.

This function can serve as a kernel for Theorem 1.

If we start once again with the Fourier cosine transform, i.e. take $\pi^{1 / 2} k_{0}(x)=2^{1 / 2} \cos x$, and then write $K_{1}(s)=K_{0}(s) /(1-s)$ we find that 
$a=0$ and $b=1$ in Theorem 1 . We then obtain what is essentially equivalent to the Fourier sine transform

Many other examples of kernels which can be used in Theorem 1 can be obtained by writing $k_{0}(x)=x^{1 / 2} J_{\nu}(x)$, where $J_{\nu}(x)$ denotes the Bessel function of order $\nu$.

The next class of kernels are those which can be used with the equations of Theorem 2. They will be designated by means of subscript two, i.e. $k_{2}(x)$ can be used in Theorem 2 . Such kernels can be generated from kernels of class one by means of convolution. The actual method of generation is embodied in the following theorem.

THEOREM 3. Let $r_{1}(x)$ and $s_{1}(x)$ be derived from the respective generalised Fourier kernels $r_{0}(x)$ and $s_{0}(x)$ according to the method described above, the constants $m$ and $n$ of (58) being the same in both cases. Then

$$
k_{2}(x)=\int_{0}^{\infty} r_{1}(u x) s_{1}(u) d u
$$

can serve as a kernel for use with Theorem 2.

Proof. We have to prove that $K_{2}(s)$ satisfies the condition imposed upon $K(s)$ in Theorem $2, \S 5$.

From the construction described above of kernels of class $k_{1}(x)$ we have

$$
R_{1}(s)=R_{0}(s) /(m+n s) \text { and } S_{1}(s)=S_{0}(s) /(m+n s),
$$

where

$$
R_{0}(s) R_{0}(1-s)=1 \text { and } S_{0}(s) S_{0}(1-s)=1
$$

and $\left|R_{0}(s)\right|$ and $\left|S_{0}(s)\right|$ are both bounded on the line $s=1 / 2+i t$.

From the boundedness of $\left|R_{0}(s)\right|$ and $S_{0}(s) \mid$ it follows from (63) that $R_{1}(s) \in L^{2}$ and $S_{1}(s) \in L^{2}$. Hence from (17), B, \$2 we have

$$
\int_{0}^{\infty} r_{1}(u x) s_{1}(u) d u=\frac{1}{2 \pi i} \int_{1 / 2-i \infty}^{1 / 2+i \infty} \frac{R_{0}(s) S_{0}(1-s)}{(m+n s)(m+n-n s)} x^{-8} d s .
$$

This shows that the integral of (61) exists and that

$$
K_{2}(s)=H_{2}(s) /\{a+b s(1-s)\}
$$

where $a=m^{2}+m n, b=n^{2}$ and

$$
H_{2}(s)=R_{0}(s) S_{0}(1-s) .
$$

From (63) it follows that 


$$
H_{2}(s) H_{2}(1-s)=1
$$

and from the boundedness of $R_{0}(s)$ and $S_{0}(s)$ it follows that $H_{2}(s)$ is bounded on the line $s=1 / 2+i t$. Thus $K_{2}(s)$ satisfies all the requirements imposed upon $K(s)$ in Theorem 2 . Consequently $k_{2}(x)$, as defined by (61), can be used as a kernel in the integral transforms of Theorem 2.

Evidently many examples of kernels of class $k_{2}(x)$ can be found by means of this theorem.

This classifisation can be continued. Let $H(s)$ be bounded on the line $s=1 / 2+i t$ and satisfy the functional equation $H(s) H(1-s)=1$ and let $P\{s(1-s)\}$ denote a polynomial in powers of $s(1-s)$ of degree $\nu$. Then kernels of class $2 \nu, k_{2 \nu}(x)$, would have Mellin transforms $H(s) / P\{s(1-s)\}$ and kernels of class $2 \nu+1, k_{2 v+1}(x)$, would have Mellin transforms $H(s) /[P\{s(1-s)\}(m+n s)]$, where $m$ and $n$ are constants. The integral transforms these kernels give rise to become exceedingly intricate as $\nu$ increases in value. For $k_{2 v}(x)$ the transform equations are symmetrical, as in Theorem 2, and each equation will contain $2 \nu$ terms in which there will be progressively increasing differentiation with respect to $u$ and $x$. For $k_{2 v+1}(x)$ the transform equations are unsymmetrical, as in Theorem 1 , the first equation will contain $2 \nu+1$ and the second $2 \nu+2$ terms. As in the even case, differentiations of increasing order occur as we progress from one term of the equation to the next.

\section{REFERENCES}

1. E. C. Titchmarsh, Theory of fourier integrals, Oxford University Press.

2. G. H. Hardy and E. C. Titchmarsh, A class of Fourier kernels, Proc. London Math. Soc. (2) vol. 35 (1933) pp. 116-155.

3. G. N. Watson, General transforms, Proc. London Math. Soc. (2) vol. 35 (1933) pp. 156-199.

4. R. E. A. C. Paley and N. Wiener, Fourier transforms in the complex domain. Amer. Math. Soc. Colloquium Publications, vol. 19, 1934.

McGill UNIVERSITY 